\title{
模拟退火及紫外光谱法用于 氨基酸多组分分析*
}

李志良 曾鸽鸣王树信 梁本喜木村敏 ${ }^{(1)}$ 吉田英 ${ }^{(1)}$

(湖南大学化学化工系, 长沙 410082; (1) 日本国立丰桥技科大学, 丰桥于 441)

\section{关键词模拟退火算法 多元分析 氨基酸 光谱分辩}

模拟退火 (SA) 系寻找全局最优并能跨越局部最优的随机优化算法, 它源于对高温物质的 退火过程的模拟即在给定温度下对微观粒子 (如原子) 平衡的统计力学模拟. $\mathrm{SA}^{[1]}$ 采用模拟 算法及随机抽样; Kirkpatrick 等 ${ }^{[2]}$ 深人研究了组合优化问题; Bohackevsky 等 ${ }^{[3]}$ 提出了通用模 拟退火 (GSA) 法; Kalivas 等 ${ }^{[4,5]}$ 研究了 GSA 用于多元校正. 本文将 SA 与 GSA 用于多组分 分析, 获得良好效果.

\section{1 原理与方法}

\section{1 退火过程及模拟}

固体物质处于熔融状态, 所组成的微观粒子处于完全随机排列组合, 以足够低的降温速率 退火, 维持体系在各温度下微粒达平衡, 则体系服从 Boltzmann 分布:

$$
f_{\mathrm{i}}=g_{\mathrm{i}} \exp \left(-E_{\mathrm{i}} / T_{\mathrm{i}}\right)=[1 / h(\mathrm{i})] \exp \left(-E_{\mathrm{i}} / b T_{\mathrm{i}}\right),
$$

此处 $i$ 指状态, $T_{i}$ 为温度, $E_{\mathrm{i}}$ 为能量, $g_{\mathrm{i}}$ 为常数, $h(\mathrm{i})$ 为分函数, $b$ 为 Boltzmann 常数; $f_{\mathrm{i}}$ 为出现 概率. 随温度降低, 高能状态出现概率越来越小, 最终趋于零. 微粒状态按 Boltzmann 分布趋 于能量最低的状态即基态. 用 Monte Carlo 模拟给定温度下微粒达热平衡的过程, 对微粒当 前态随机地进行微扰. 令 $E_{\mathrm{a}}$ 和 $E_{\mathrm{b}}$ 分别为当前和微扰新状态的能量, 能差为 $\Delta E=E_{\mathrm{b}}-E_{\mathrm{a}}$. 若 $E_{\mathrm{a}}>E_{\mathrm{b}}$, 则无条件接受新状态并代替当前态; 若 $E_{\mathrm{a}} \leqslant E_{\mathrm{b}}$, 则此概率 $\exp \left[-\left(E_{\mathrm{b}}-E_{\mathrm{a}}\right) / T\right]$ 接受此不利 状态为当前态. 继续徐徐降温并重复抽样, 直至获最低能量状态.

\section{2 模拟算法}

将状态对应於待优化参数 $x$; 对能量 $E$ 相当于目标函数 $J$; 视退火温度 $T$ 与 Boltzmann 常 数为控制随机搜索程度的参数 $B$. 具体步骤如下: (1) 设定初始态 $x_{\mathrm{a}}$ 并计算其目标函数 $J_{\mathrm{a}}=J\left(x_{\mathrm{a}}\right)$. (2) 施加随机微扰得新状态 $x_{\mathrm{b}}$, 计算 $J_{\mathrm{b}}=J\left(x_{\mathrm{b}}\right)$ 及能差 $\Delta J=J_{\mathrm{b}}-J_{\mathrm{a}}$. (3) 比较 $J_{\mathrm{a}}$ 与 $J_{\mathrm{b}}$, 若 $J_{\mathrm{a}}>J_{\mathrm{b}}$, 则 无条件接受 $x_{\mathrm{b}}$ 为当前态, 若 $J_{\mathrm{a}}<J_{\mathrm{b}}$, 则算概率 $f=\exp (-B \Delta J)$. (4) 产生一均匀随机数 $u \in[0,1]$; 若 $f \geqslant u$, 则接受 $x_{\mathrm{b}}$ 并作为当前态, 否则拒绝. (5) 重复微扰直至抽样达稳定即收玫得全局最优 解. (6) 若拒绝则重新依上计算. 适择控制参数 $B^{120}$ 便可达全局最优.

1994-04-20 收稿, 1994-12-14 收修改稿

*日本政府文部省与国家教委及国家自然科学基金资助课题 


\section{3 通用算法}

将 $J=J(x)$ 定义为多维连续函数, 并寻优得某状态 $x_{0}$ 使 $J\left(x_{0}\right)$ 达最优. 设当前态 $x_{\mathrm{a}}$, 微扰产 生新随机态

$$
x_{\mathrm{b}}=x_{\mathrm{a}}+v \cdot s,
$$

$v$ 为方向矢量, $s$ 为变化步长. 任意产生 $n$ 个 $N(0,1)$ 随机数 $W_{\mathrm{i}}(\mathrm{i}=1,2, \cdots, n)$, 计算其余弦方向 $v_{\mathrm{i}}=w_{\mathrm{i}} / \sqrt{\Sigma w_{\mathrm{i}}^{2}}$, 则状态为

$$
x_{\mathrm{b}}=x_{\mathrm{a}}+s v \Rightarrow x_{\mathrm{bi}}=x_{\mathrm{ai}}+v_{\mathrm{i}} \cdot s .
$$

GSA 取消了 SA 改变 $B$ 的控温循环, 并修正接受概率:

$$
f=\exp \left[-B \cdot \Delta J \cdot(D J)^{a}\right]=\exp [-B \cdot \Delta J / D J],
$$

式中 $B$ 仍为控制因子, $\Delta J=E_{\mathrm{b}}-E_{\mathrm{a}}$ 为微扰能差, $D J=E_{\mathrm{a}}-E_{\mathrm{b}}$ 为相对能差, $a<0$ 为任意负值, 当 $a=0$ 时 GSA 还原为 SA. 常取 $a=-1$, 则接受概率降低, 有利收玫. 为搜索接近全局最优时 应不再接受不利状态 (即接受概率近零). 步长 $s$ 在 GSA 中通常不变, 也可变化》。本文逐步 降低步长 $s$, 以提高优化精度. $J_{0}$ 为全局最优点的目标函数, 若已知可直接选取; 若未知或难 预先确定, 则可先尝试某值再据运算予调整. 故 GSA 提高了优化精度和收玫速度.

\section{4 控制参数 $B$}

曾有建议 ${ }^{[3]}$ 适选 $B$ 值使接受概率 $f \in[0.5,0.90]$, 常使 $f=0.8$. 若选择不当则或使算法 $f$ 过 低而陷人局优; 或使算法 $f$ 过高而难於收玫, 即完全随机游走.

\section{5 多元校正}

最常见的多组分光谱分析定量模型参见文献[8]. 由纯组分直接测定或标准样处理间接确 定量测吸光系数.

\section{6 多元定量}

测量未知试样的吸收光谱数据可依 SA 及 GSA 法预测未知浓度, 即实现多组分同时定量 ${ }^{[8]}$.

\section{2 实验部分}

\section{1 仪器与试剂}

日本岛津 UV-240 和美国 Beckman DU-7HS 及国产上海 752 型紫外可见分光光度计; IBM-PC/AT 386 微机及兼容机; VAX/DMS 工作站用於编制运行 SA 和 GSA 及扫描算法程 序. 色氨酸 $\mathrm{Trp}$, 酪氨酸 Tyr, 苯丙氨酸 Phe, 及 3,4-二羟基苯丙氨酸 Dhp 等均为色谱纯试剂 (Merck), 用常规法配成 $0.1 \mathrm{~mol} / \mathrm{L} \mathrm{HCl}$ 储备液并稀释为工作液, 其它试剂均为 $\mathrm{AR}$ 以上.

\section{2 实验方法}

配成纯组分标液和多组分混液测量紫外光谱, 扫描波长范围为 $190 \sim 300 \mathrm{~nm}$, 狭缝宽度为 $1.0 \mathrm{~nm}$, 在 $200 \sim 290 \mathrm{~nm}$ 范围内间隔 $1 \mathrm{~nm}$ 读取数据输人计算机处理.

\section{3 结果与讨论}

\section{1 紫外光谱}

四组分氨基酸光谱 (图 1) 重叠严重, 须予分离定量或作多元分辨, 方能实现其多组分分析.

\section{2 算法参数影响}

以对称函数 $f\left(x_{1}, x_{2}\right)=x_{1}^{2}+2\left(1-\cos 2 \pi x_{1}\right)+3 x_{2}^{2}+4\left(1-\cos 4 \pi x_{2}\right)$ 作为目标函数为例, 考察 GSA 
中循环步长 $s$ 及控制参数 $B$ 对求解精度的影 响. 该函数有若干局部最优点和零值全局最 优点 $\left(x_{1}=0, x_{2}=0\right)$. 假定优化约束区域为 $-1 \leqslant x\left(x_{1}, x_{2}\right) \leqslant 1$, 任取点 $\left(x_{1}=1, x_{2}=1\right)$ (实为 最远者的四点之一) 为初始点作最优搜索. 取 $B=1.0$, 步长固定为 $s=0.2$, 经 3 次寻优得 目标函数即搜索误差分别为 $7.3 \%, 4.2 \%$, $10.5 \%$; 改变搜索步长 (以半幅收缩), 仍取 $B=1.0$, 步长初值为 $s=0.2$, 以半幅收缩, 经 3 次寻优搜索得目标函数为 $0.0720 \%, 0.0380 \%$, $0.0029 \%$ ．表明引人收缩步长后, 其优化求 解精度大大改善. 参数 $B$ 对求解结果亦有 较大影响, 若 $B$ 过小 (如 $B=0.01), f$ 将过大,

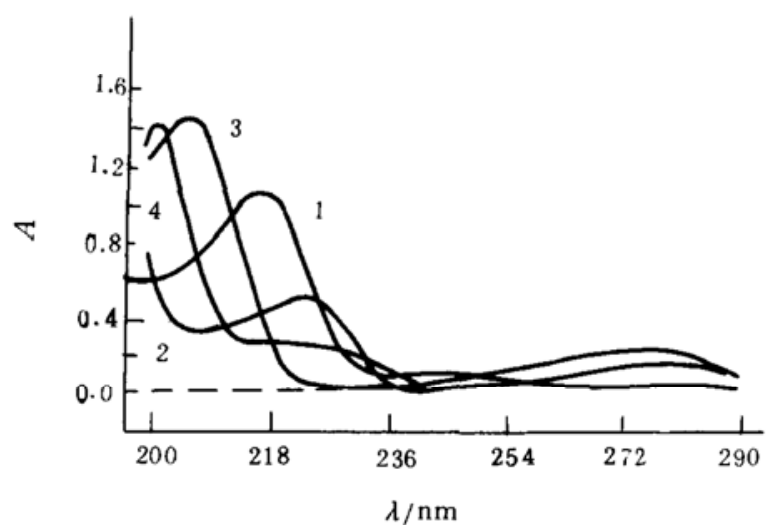

图 1 紫外光谱

氨基酸： 1 为 Trp, 2 为 Tyr, 3 为 Phe, 4 为 Dhp. 介质为 0.1 $\mathrm{mol} / \mathrm{L} \mathrm{HCl}$ 抽样不能收玫; 若 $B$ 太大 (如 $B=100$ ), 则 $f$ 将太小, 算法易陷人局部最优而得不到全局最优. 通常算法受到许多因素的制约, 比如局部最优点数、函数形状、变化缓急等. 欲使算法在极值 点附近搜索实际上也难於实现. 为保证能搜索整个区域, 步长 $s$ 宜取稍大. 对有约束优化, 步 长宜取约为区域 $10 \%$ 左右. 倘欲改善精度,则步长可逐渐收缩.

表 1 四组分混合复基酸试样同时测定结果

\begin{tabular}{|c|c|c|c|c|c|c|c|c|c|c|c|c|c|c|c|c|}
\hline \multirow{3}{*}{$\begin{array}{l}\text { 试 } \\
\text { 样 } \\
\text { 号 }\end{array}$} & \multicolumn{4}{|c|}{ 加人量 Added $\left(\times 10^{-6}\right)$} & \multicolumn{12}{|c|}{ 测得量 Found $\left(\times 10^{-6}\right.$ 上排数据) 及回收率 Recovery $/ \%$ (下排数据 ) } \\
\hline & \multirow[b]{2}{*}{ Tyr } & \multirow[b]{2}{*}{$\operatorname{Trp}$} & \multirow[b]{2}{*}{ Phe } & \multirow[b]{2}{*}{ Dhp } & \multicolumn{4}{|c|}{ 卡尔滤波 } & \multicolumn{4}{|c|}{ 因子分析 } & \multicolumn{4}{|c|}{ 本法 GSA } \\
\hline & & & & & Tyr & Trp & Phe & Dhp & Tyr & Trp & Phe & Dhp & Tyr & Trp & Phe & Dhp \\
\hline \multirow[t]{2}{*}{1} & 4.670 & 5.180 & 14.33 & 8.280 & 4.570 & 5.127 & 14.01 & 8.274 & 4.501 & 5.214 & 14.572 & 8.532 & 4.568 & 5.126 & 14.012 & 8.276 \\
\hline & & & & & 97.85 & 98.97 & 97.76 & 100.8 & 96.4 & 100.7 & 101.7 & 103.9 & 97.82 & 98.96 & 97.78 & 100.82 \\
\hline \multirow[t]{2}{*}{2} & 3.736 & 6.216 & 23.89 & 4.104 & 3.734 & 6.137 & 23.43 & 4.116 & 3.716 & 6.225 & 23.792 & 4.306 & 3.726 & 6.231 & 23.401 & 4.126 \\
\hline & & & & & 99.95 & 98.73 & 98.05 & 100.3 & 99.5 & 100.1 & 99.6 & 104.9 & 99.73 & 100.24 & 97.95 & 100.54 \\
\hline \multirow[t]{2}{*}{3} & 3.736 & 4. 144 & 33.45 & 12.31 & 3.561 & 4.159 & 32.71 & 12.43 & 3.422 & 4.225 & 32.600 & 12.872 & 3.561 & 4.168 & 32.70 & 12.54 \\
\hline & & & & & 95.32 & 100.4 & 97.80 & 100.9 & 91.6 & 101.9 & 97.5 & 104.6 & 95.32 & 100.58 & 97.76 & 100.87 \\
\hline \multirow[t]{2}{*}{4} & 8.406 & 2.072 & 23.89 & 6.156 & 8.315 & 2.072 & 24.74 & 6.428 & 8.161 & 2.161 & 23.953 & 6.626 & 8.308 & 2.072 & 24.47 & 6.428 \\
\hline & & & & & 98.92 & 100.0 & 103.5 & 104.4 & 97.1 & 104.3 & 100.3 & 107.6 & 98.92 & 100.0 & 102.43 & 104.4 \\
\hline \multirow[t]{2}{*}{5} & 7.472 & 1.036 & 9.556 & 16.42 & 7.319 & 1.045 & 9.998 & 16.428 & 7.086 & 1.131 & 9.732 & 16.965 & 7.149 & 1.045 & 9.978 & 16.428 \\
\hline & & & & & 97.96 & 100.9 & 104.6 & 100.9 & 94.6 & 109.2 & 101.8 & 103.3 & 95.68 & 100.9 & 104.42 & 100.9 \\
\hline \multirow[t]{2}{*}{6} & 6.538 & 2.072 & 4.778 & 10.26 & 6.530 & 2.026 & 4. 516 & 10.34 & 6.500 & 2. 125 & 5.014 & 10.341 & 6.530 & 2.026 & 4.512 & 10.342 \\
\hline & & & & & 99.88 & 97.78 & 94.51 & 100.8 & 99.4 & 102.6 & 104.9 & 100.8 & 99.88 & 97.78 & 94.32 & 100.8 \\
\hline \multirow[t]{2}{*}{7} & 5.604 & 5.180 & 14.33 & 2.052 & 5.646 & 5.060 & 13.72 & 2.146 & 5.689 & 5.167 & 14.646 & 2. 164 & 5.649 & 5.060 & 13.709 & 2.146 \\
\hline & & & & & 100.8 & 97.68 & 95.75 & 104.6 & 101.5 & 19.7 & 102.2 & 105.4 & 100.80 & 97.7 & 95.67 & 104.6 \\
\hline \multirow[t]{2}{*}{8} & 2.902 & 4. 144 & 23.89 & 12.31 & 2.774 & 4.075 & 23.62 & 12.40 & 2.783 & 4.202 & 23.954 & 12.612 & 2.774 & 4.070 & 23.62 & 12.526 \\
\hline & & & & & 99.00 & 98.35 & 98.86 & 100.7 & 99.3 & 101.4 & 100.3 & 102.5 & 99.0 & 98.9 & 98.9 & 101.75 \\
\hline \multirow[t]{2}{*}{9} & 2.802 & 4. 144 & 23.89 & 12.31 & 2.871 & 4.058 & 23.58 & 12.41 & 2.893 & 4. 187 & 24.105 & 12.436 & 2.872 & 4.054 & 23.456 & 12.412 \\
\hline & & & & & 102.5 & 97.93 & 99.14 & 100.8 & 103.3 & 101.0 & 100.9 & 101.0 & 102.5 & 97.83 & 98.18 & 100.8 \\
\hline \multirow[t]{2}{*}{10} & 4.670 & 3.108 & 23.89 & 2.052 & 4.890 & 3.021 & 24.43 & 1.995 & 4.941 & 3.005 & 24.702 & 1.986 & 4.890 & 3.021 & 24.507 & 1.989 \\
\hline & & & & & 104.7 & 97.22 & 102.3 & 97.23 & 105.8 & 96.7 & 103.4 & 96.8 & 104.7 & 97.22 & 102.58 & 96.93 \\
\hline
\end{tabular}




\section{3 四组分氨基酸同时测定}

对四组分氨基酸混合体系的光谱数据处理, 参数 $B$ 的选择无规可循且取值无界; 步长 $s$ 选取亦无经验可言但区域有下限, 即约束条件 $x_{\mathrm{i}} \geqslant 0$; 我们依据测试光谱数据确定各组分浓度 可能上限, 然后以此区域边界取初始步长 (约为 $1 / 10$ ) 求解. 均经百次试算初始不接受不利状 态的概率 $P$, 若 $P \geqslant 0.9$ 或 $P \leqslant 0.5$, 则返回重设参数. 以最小二乘准则依 GSA 预测未知试样中 各氨基酸组分的浓度, 与卡尔曼滤波 $(\mathrm{KF})^{[8,9]}$ 及目标因子分析 $(\mathrm{FA})$ 求解比较, 结果相互一致, 优化精度与预测结果良好: 回收率为 $95.3 \% \sim 104.6 \%$.

\section{4 初步结论}

GSA 与 SA 系有用的化学计量学新算法, 用於同时光谱测定, 为氨基酸分析提供了一种 优良的和有效的新方法. 也可望在其它方面取得广泛应用.

\section{参考文献}

1 Metropolis N, Rosenbluth A, Rosenbluth $\mathrm{M}$ et al. Equation of state calculations by fast computing machines. J Chem Phys, 1953, 21: 1087 1 092

2 Kirkapatrick S, Gelatt Jr C D, Vecchi M P. Optimization by simulated annealing. Science, 1983, 220:671 680

3 Bohackersky I O, Jahnson M E, Stein M L. Generalized simulated annealing for function optimization. Technom, 1986, 28(3): 209 217

4 Kalivas J H, Roberts N, Sutter J M. Global optimization by simulated annealing with wavelength section for ultraviolet-visible spectrophotometry. Anal Chem, 1989, 61:2024 2203

5 Kalivas J H. Generalized simulated annealing for calibration sample selection from an existing set and orthogonalization of undesigned experiments. J Chemom, 1991, 5(1):37 48

6 Collins N E, Eglese R W, Golden B L. Simulated annealing-an annotated bibliography. Am J Math Mang Sci, 1988, 8(3/4): $209 \sim 307$

7 Vanderbilt D, Louie S G. A Monte Carlo simulated annealing approach to optimization over continuous variables. J Comput Phys, 1984, 56:259 271

8 Li Z L, Shi L M, Li M L et al. A highly sensitive and selective method for trace multicomponent analysis. Acta Chim Sin, 1990, 48(11): $1101 \sim 1107$

9 Shi L M, Xu Z H, Li Z L et al. Application of the Kalman filter algorithm to the simultaneous determination four amino acids by direct uv spectrophyotometry. J Micronutr Anal, 1990, 8:1 12 